\title{
SECULAR EVOLUTION OF CATACLYSMIC VARIABLES WITH IRRADIATION-INDUCED MASS TRANSFER
}

\author{
H. RITTER ${ }^{1}$, Z. ZHANG ${ }^{1}$, J. M. HAMEURY ${ }^{2}$ \\ 1. MPI für Astrophysik, D-85748 Garching, Germany \\ 2. Observatoire de Strasbourg, F-6700 Strasbourg, France
}

\section{Introduction}

The possible importance of the reaction of a low-mass star to external irradiation for the long-term evolution of compact binaries has been noted only rather recently; first in the context of the evolution of low-mass X-ray binaries (e.g. Podsiadlowski 1991; Harpaz \& Rappaport 1991; Frank, King \& Lasota 1992; Hameury et al. 1993) and subsequently by Ritter, Zhang \& Kolb (1995a,b, hereafter RZK) also for the evolution of cataclysmic variables (CVs). Based on a simple model for describing the reaction of a lowmass star to irradiation RZK showed that CVs can be dynamically unstable against irradiation-induced mass transfer and that, as a consequence of this, mass transfer could occur via cycles in which phases of high, irradiationenhanced mass transfer alternate with phases of little or no mass transfer. The occurrence of such mass transfer cycles in CVs was subsequently discussed from a more general point of view by King (1995) and King et al. (1995). Whereas the possibility of mass transfer cycles in CVs is now fully recognised, the question as to which systems can undergo such cycles and which cannot has not yet been addressed in detail. It is the purpose of this contribution to provide at least a partial answer to this question.

\section{Stability against irradiation-induced mass transfer}

The criterion for dynamical stability against irradiation-induced mass transfer as derived by RZK is

$$
\zeta_{\mathrm{s}}-\zeta_{R}>\zeta_{\mathrm{irr}}=-M_{\mathrm{s}} \frac{\partial}{\partial \dot{M}_{\mathrm{s}}}\left(\frac{\partial \ln R_{\mathrm{s}}}{\partial t}\right)_{\mathrm{irr}} .
$$

Here $M_{\mathrm{s}}, R_{\mathrm{s}}, \dot{M}_{\mathrm{s}}$ and $\zeta_{\mathrm{s}}$ are respectively the mass, the radius, mass loss rate and adiabatic mass radius exponent of the secondary (the mass donor). $\zeta_{R}$

$$
449
$$

A. Evans and J. H. Wood (eds.), Cataclysmic Variables and Related Objects, 449-452.

(C) 1996 Kluwer Academic Publishers. Printed in the Netherlands. 
is the mass radius exponent of the critical Roche radius and $\left(\partial \ln R_{s} / \partial t\right)_{\text {irr }}$ the thermal relaxation term due to irradiation.

In the following we shall first discuss the consequences of (1) in the framework of the simple model adopted by RZK in which it is assumed that a fraction $s$ of the secondary's surface is exposed to a uniform external irradiation flux $F_{\text {irr. }}$. Combining equations (5) and (11) of RZK, (1) can be rewritten as follows:

$$
C=2\left(\zeta_{\mathrm{s}}-\zeta_{R}\right) \frac{\tau_{\mathrm{KH}, 0}}{\tau_{\mathrm{M}}} \mathcal{F}^{-1}>2 s x g(x),
$$

where $\tau_{\mathrm{KH}, 0}=\frac{G M_{s}^{2}}{R_{s} L_{s, 0}}$ is the Kelvin-Helmholtz time of the unirradiated star, $\tau_{\mathrm{M}}=-M_{\mathrm{s}} / \dot{M}_{\mathrm{s}}$ the mass transfer time-scale and $\mathcal{F}$ a function which scales roughly as the inverse of the relative mass of the secondary's convective envelope. In the bipolytrope approximation (Kolb \& Ritter 1992) $\mathcal{F}=\{\}\{\}^{-1}$, where the expressions in the curly brackets are defined in equation (10) of RZK. Furthermore, in (2), $x=F_{\mathrm{irr}} /\left\{\sigma T_{0}^{4}\right\}$ is the dimensionless irradiating flux, $T_{0}$ being the effective temperature of the unirradiated star, and

$$
g=-\frac{\partial}{\partial F_{\mathrm{irr}}}\left[\sigma T_{\mathrm{irr}}^{4}-F_{\mathrm{irr}}\right]=-\frac{\partial}{\partial x}\left[\left(\frac{T_{\mathrm{irr}}}{T_{0}}\right)^{4}-x\right]=g(x),
$$

where $T_{\text {irr }}$ is the effective temperature on the irradiated parts of the star. The virtue of rewriting (1) in the form of (2) is that in (2) terms which depend on irradiation (right hand side) are separated from terms which do not (left hand side). In fact, the function $C$ defined in (2) depends only on binary parameters and via $\tau_{\mathrm{M}}$ on the adopted model for the long-term evolution. Thus, along an evolutionary sequence the value of $C$ is always known. The question of whether (2) is fulfilled or not thus depends on the value of $2 s x g(x)$.

\section{Reaction of the stellar surface to external irradiation}

Using a simple one-zone model for the superadiabatic surface layer of lowmass stars, RZK derived an explicit expression for $g$ (their equation 7 ). Here we shall not make use of this result because it does not apply to the whole mass range of interest. Rather we shall base our discussion on very general properties of the function $g$ and on results of numerical calculations of $g$ performed using the code described by Hameury et al. (1993).

First of all it is important to notice the following general properties of the function $g(x)$ which are entirely model independent:

(ii) $g(x=0) \leq 1$ for positive albedos, and

$$
\lim g(x \rightarrow \infty)=0 \text {. }
$$


Because of these properties, $g(x)$ must be a rather steeply decreasing function of $x$ with $0<g(x) \ll 1$ if $x>$ a few. This is true, in particular, for stars with a mass $M \lessgtr 0.6 \mathrm{M}_{\odot}$, for which $g(x=0) \approx 1$. As a consequence, the function $x g(x)=0$ for $x=0$ and $x \rightarrow \infty$, and $x g(x)$ attains a maximum $\operatorname{Max}[x g(x)] \approx 0.5$ for values $x \approx 1$ to a few. This holds in the whole mass range of interest, i.e. $0.1 \lesssim M / \mathrm{M}_{\odot} \lesssim 1$. Based on these results we can now resume the discussion of (2).

\section{Secular evolution of CVs with irradiation}

Violation of (2) is only a necessary but not a sufficient condition for the occurrence of mass transfer cycles. On the other hand, fulfillment of (2) is a sufficient condition for the absence of mass transfer cycles. Now, because the function $x g(x)$ has a maximum value, we can state that no mass transfer cycles will occur if

$$
C>2 s \operatorname{Max}[x g(x)] \approx s .
$$

So far, we have carried out our discussion in the framework of the simple model of RZK. However, even if we adopt a much more realistic and elaborate model, i.e. the point source model, in which the secondary is assumed to be irradiated by a point source at the location of the white dwarf primary, an analogous argument can be made to yield [in place of (7)]

$$
C>\operatorname{Max}\left[\int_{0}^{\theta_{\max }} x(\theta) g\{x(\theta)\} \sin \theta d \theta\right],
$$

where now the irradiating flux $F_{\text {irr }}$ depends on $\theta$, the colatitude of a given point on the stellar surface as measured from the substellar point. Using numerical results for $g(x)$ we find the maximum of the integral in (8) to be smaller than $2 s \operatorname{Max}[x g(x)]$ by about a factor of 2 if $s \approx 1 / 2$. This is clearly seen in Fig. 1 where we plot as a full line the run of $C$ as a function of $M_{\mathrm{s}}$ for a standard $\mathrm{CV}$ evolution $\left(M_{1}=1 \mathrm{M}_{\odot}, M_{2, \mathrm{i}}=1 \mathrm{M}_{\odot}\right.$, disrupted magnetic braking model), the line corresponding to $2 s \operatorname{Max}[x g(x)]$ for $s=1 / 2$ (dashed line) and the right hand side of (8) as a dash-dotted line. Eq. (8) and Fig. 1 lead to the conclusion that in the framework of standard CV evolution no mass transfer cycles can occur if $M_{\mathbf{s}} \lesssim 0.7 \mathrm{M}_{\odot}$. The reason for the stability of systems with $M_{\mathrm{s}} \lessgtr 0.7 \mathrm{M}_{\odot}$ is that the function $C$ increases strongly with decreasing $M_{\mathrm{s}}$ whereas the right hand sides of (7) and (8) remain almost constant. The strong increase of $C$ with decreasing $M_{\mathrm{s}}$ has two main reasons: first, with decreasing $M_{\mathrm{s}}$ the stellar luminosity decreases steeply (mass-luminosity relation!), i.e. $\tau_{\mathrm{KH}, 0}$ increases correspondingly; second, with decreasing $M_{\mathrm{s}}$ the factor $\mathcal{F}$ grows strongly because the (relative) mass of the convective envelope grows until the star becomes fully convective. In other words, $C$ reflects the thermal inertia of the convective envelope 


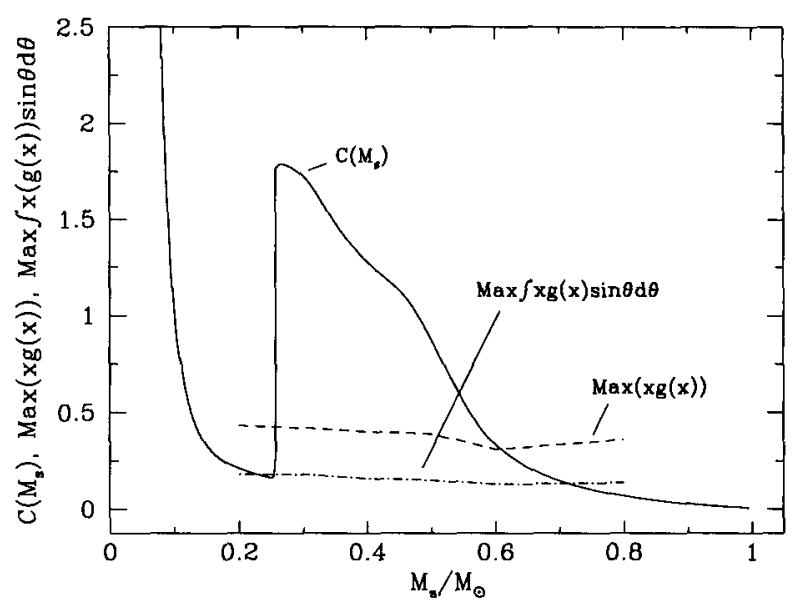

Figure 1. The run of $C, \operatorname{Max}[x g(x)]$ and $\operatorname{Max}\left[\int x g(x) \sin \theta d \theta\right]$ as a function of the secondary's mass $M_{8}$. For details see text.

which increases with decreasing $M_{\mathrm{s}}$ because its heat capacity increases and the luminosity available for changing the entropy of the envelope decreases.

In principle there are two possibilities for reducing the value of $C$ and thus widening the mass range in which mass transfer cycles are possible. However, both these possibilities are not compatible with standard CV evolution. The first possibility consists of increasing $\tau_{\mathrm{M}}$, i.e. of reducing the secular mean mass transfer rate. However, doing so by a significant factor would destroy the period gap, the width of which sets an upper limit of $\tau_{\mathrm{M}} \sim 10^{8} \mathrm{yr}$. The second possibility consists of reducing the value of $\zeta_{s}-\zeta_{R}$ which is possible only if a CV suffers significant consequential angular momentum loss. The difficulties arising from such a proposition have been examined in detail by King \& Kolb (1995).

\section{References}

Frank, J., King, A.R., Lasota, J.P., 1992, Ap. J., 385, L45

Hameury, J.M., King, A.R., Lasota, J.P., Raison, F., 1993, A\&A, 277, 81

Harpaz, A., Rappaport, S., 1991, Ap. J., 383, 739

King, A.R., 1995, in "Cataclysmic Variables", eds A. Bianchini et al., Kluwer, p523

King, A.R., Kolb, U., 1995, Ap. J., 439, 330

King, A.R., Frank, J., Kolb, U., Ritter, H., 1995, Ap. J., 444, L37

Kolb, U., Ritter, H., 1992, A\&A, 254, 213

Podsiadlowski, P., 1991, Nature, 350, 136

Ritter, H., Zhang, Z., Kolb, U., 1995a, IAU Symp. No. 165, ed. E.P.J. van den Heuvel, in press

Ritter, H., Zhang, Z., Kolb, U., 1995b, in "Cataclysmic Variables", eds A. Bianchini et al., Kluwer, p479 